\title{
ENTROPY TRANSPORTATION EQUATION BASED ON LOSS MECHANISM ANALYSIS WITH HIGH FIDELITY TURBULENCE SIMULATION RESULTS
}

\author{
Hui Li \\ Department of Energy and Power Engineering, \\ Tsinghua University \\ hui-li17@mails.tsinghua.edu.cn \\ Beijing, China
}

\author{
Xin Yuan \\ Department of Energy and Power Engineering, \\ Tsinghua University \\ yuanxin@mail.tsinghua.edu.cn \\ Beijing, China
}

\author{
Xinrong Su* \\ Department of Energy and Power Engineering, \\ Tsinghua University \\ suxr@mail.tsinghua.edu.cn I \\ Beijing, China
}

\begin{abstract}
The entropy analysis method based on the second law of thermodynamics has been widely used to evaluate the sources of irreversibility in components and systems, but this method is limited to global analysis. The local entropy production method can accurately calculate the sources of energy loss and its location, which is beneficial to improve the performance and efficiency of apparatus. According to the time-averaging the transport equation for entropy, the intrinsic relationship between irreversible energy loss and entropy production is found, and the mechanisms of entropy production contain four terms, which are dissipation in a mean and fluctuating velocity field and heat flux in a mean and fluctuating temperature field respectively. Since the real flow is unsteady turbulent flow, the contribution of unsteady fluctuating terms in entropy production cannot be ignored, and the amount of the unsteady fluctuating terms is also the key to improve the performance of components. Combined with the hybrid RANS/LES sim ulation method, a linear shroudless rotor with flat tip is analysed by local entropy production. For high fidelity turbulence simulation results, the four different mechanisms of entropy production are obtained. It is found that fluctuating dissipation is accounts about $30 \%$ of the total local entropy generation where the tip leakage flow occurs. Due to the existence of tip leakage vortex, passage vortex and the interaction of such complex vortices, the local turbulence intensity is enhanced. The local entropy production method can effectively analyse the loss mechanism and its distribution of turbulent flow, especially the contribution of unsteady fluctuating in entropy production.
\end{abstract}

\section{INTRODUCTION}

From the perspective of thermodynamics, the loss in flow field is caused by the irreversibility of the flow. In the design, the total pressure loss coefficient, the energy loss coefficient, the enthalpy loss coefficient, etc. are often used as an evaluation index for estimating the magnitude of the loss. These loss coefficients are simply global values that reflects a sum or result of all losses in system and cannot provide detailed information on the location of flow loss. Denton pointed out that the total loss coefficient and the total pressure loss coefficient are not ideal for measuring the loss in the turbine (Denton, 1993). It is recommended to use the entropy loss coefficient. Combined with the laws of thermodynamics and entropy transportation equation, it is a good idea to use entropy production to measure loss in flow field, which can solve the above mentioned problems.

In recent years, the use of entropy production to analyse the loss in heat transfer equipment has been applied successfully, and this method is getting more and more attention in other research area. Under the condition of no chemical reaction, no internal heat source, and only considering the flow and heat transfer process, Bejan and Kestin proposed the concept of minimum entropy production during flow heat transfer for the first time (Bejan and Kestin, 1983). Kock and Herwig performed time-averaging process on the transport equation for entropy of incompressible fluid, and classified the entropy production (Kock and Herwig, 2004). The presented model has been modified according to the results of direct numerical simulations and it can be 
easily implemented in CFD post-process (Kock and Herwig, 2005). Chu and Liu analysed a high-temperature confined jet by entropy generation and verified the main factors of irreversible loss (Chu and Liu, 2009). Jin and Herwig used direct numerical simulation method to analyse the physical mechanism of the influence of wall roughness on turbulence by entropy generation method (Jin and Herwig, 2014). It can be seen that the entropy generation rate is a good quantitative indicator for calculation local loss, and the total loss can be obtained by volume integral. However, the reliability of this method is highly dependent on the accuracy of numerical calculations. The ideal entropy generation rate prediction requires the CFD model to predict flow process correctly, especially with mixing and separation. Therefore, the selection of the numerical method is also a key factor in determining whether the loss results are credible.

With the continuous development of Computational Fluid Dynamics (CFD), many realistic and complicated flows can be simulated by numerical calculation, and the numerical simulation results can predict flow regime correctly. Although the Reynolds Average Navier-Stokes (RANS) method is widely used, it carries out more modelling for the flow field. When there are strong shear, unsteadiness and large separation flows, the credibility of entropy generation rate results by RANS is very low. Currently, high fidelity simulation methods are gradually being applied, which include Direct Numerical Simulation (DNS), Large Eddy Simulation (LES) and Delayed Detached Eddy Simulation (DDES). DNS is directly solving the NavierStokes equation without assumptions, and the entropy generation rate is the most reliable. However, DNS requires a lot of computational resources for complex flows, so this method is mainly applied to study the mechanism of some basic cases such as shock wave boundary layer interference on plate (Lee, Malaya, et al., 2015. Yang, Yao, et al., 2016). LES and DDES perform well in calculation flow field, and can provide reliable entropy generation rate results comparing with RANS method. For high Reynolds number flows, LES method consumes more computing resources than DDES. From the perspective of ensuring the reliability of the results and reducing the computation time, DDES method is a good choice. Bian et al. (Bian, Lin, et al., 2017), Lin et al. (Lin, Yaun, et al., 2017. Lin, Su, et al., 2016) obtained the detailed flow field structure in a transonic cascade channel by DDES method.

The entropy generation is rarely used in turbomachinery. Lin et al. (Lin, Yaun, et al., 2017) applied the entropy generation rate to analyse a high pressure turbine. Li et al. (Li, Su, et al., 2019) conducted a preliminary analysis of tip leakage flow loss by entropy generation rate. In this paper, based on the entropy transportation and law of thermodynamics, the formula of entropy generation rate is derived in detail. The in-house multi-block parallel solver (Su and Yaun, 2016. Su and Yaun, 2010) with independent intellectual property is used to simulate a unshrouded blade with a tip gap of $1 \%$ blade height and the tip leakage flow is analysed by the entropy generation rate.

\section{TRANSPORT EQUATION FOR ENTROPY}

According to energy conservation, the internal energy equation can be obtained as following;

$$
\frac{D e}{D t}=Q-\frac{1}{\rho} \frac{\partial q_{i}}{\partial x_{i}}+\frac{\Phi}{\rho}-\frac{p}{\rho} \frac{\partial u_{i}}{\partial x_{i}} .(1)
$$

where De/Dt represents the amount of change in internal energy per unit time and mass. $Q$ represents the absorbed external heat per unit time and mass. $\partial q_{i} / \partial x_{i}$ represents the reduced heat per unit time and volume. $\Phi-\mathrm{p} \partial u_{i} / \partial x_{i}$ represents the deformation work by the stress tensor, where $\Phi$ is the dissipation function, which dissipates mechanical energy to improve internal energy, and the $-p \partial u_{i} / \partial x_{i}$ is the expansion work of the fluid, which reduces the internal work.

From the differential relationship of thermodynamic energy, the relationship between entropy production and internal energy can be obtained as shown below:

$$
T \frac{D s}{d t}=\frac{D e}{D t}+p \frac{D}{D t}\left(\frac{1}{\rho}\right) .
$$

The mass conservation equation is deformed and the formula is shown as following:

$$
\frac{D}{D t}\left(\frac{1}{\rho}\right)=\frac{1}{\rho} \frac{\partial u_{i}}{\partial x_{i}}
$$

Then the entropy transportation equation is derived by combing with the equation (1), and equation (2), and equation (3), and Fourier heat Conduction, which is shown as equation (4).

$$
\rho \frac{D s}{D t}=-\operatorname{div}\left(\frac{\vec{q}}{T}\right)+\frac{\Phi}{T}+\frac{\Phi_{\Theta}}{T^{2}}+\frac{\rho Q}{T} .
$$

when there is no internal heat source, $\rho Q / T$ is zero. The dissipation function $\Phi$ and $\Phi_{\Theta}$ are given in detail later.

\section{Time-averaging the entropy transport equation}

The Reynolds Averaged Navier Stokes approach can be used to split the instantaneous values into time-mean and fluctuating parts, such as $s=\bar{s}+s^{\prime}$. The entropy transport equation for incompressible fluid is time averaged by Reynolds Averaged Navier Stokes approach. Since this paper mainly introduces the entropy generation formula, only the derivation process of entropy generation is written here.

The entropy generation by dissipation can be written: 


$$
\begin{aligned}
& \frac{\Phi}{T}=\frac{\mu}{T}\left[2 \left\{\left(\frac{\partial\left(\bar{u}+u^{\prime}\right)}{\partial x}\right)^{2}+\left(\frac{\partial\left(\bar{v}+v^{\prime}\right)}{\partial y}\right)^{2}\right.\right. \\
& \left.+\left(\frac{\partial\left(\bar{w}+w^{\prime}\right)}{\partial z}\right)^{2}\right\}+\left(\frac{\partial\left(\bar{u}+u^{\prime}\right)}{\partial y}+\frac{\partial\left(\bar{v}+v^{\prime}\right)}{\partial x}\right)^{2} \\
& \left.+\left(\frac{\partial\left(\bar{u}+u^{\prime}\right)}{\partial z}+\frac{\partial\left(\bar{w}+w^{\prime}\right)}{\partial x}\right)^{2}+\left(\frac{\partial\left(\bar{v}+v^{\prime}\right)}{\partial z}+\frac{\partial\left(\bar{w}+w^{\prime}\right)}{\partial y}\right)\right]
\end{aligned}
$$

Then, time-averaged local entropy generation by dissipation is merged by discarding the average of the fluctuating multiplications. They read:

$$
\begin{aligned}
& \overline{\left(\frac{\Phi}{T}\right)}=\frac{\mu}{\bar{T}}\left[2\left\{\left(\frac{\partial \bar{u}}{\partial x}\right)^{2}+\left(\frac{\partial \bar{v}}{\partial y}\right)^{2}+\left(\frac{\partial \bar{w}}{\partial z}\right)^{2}\right\}\right. \\
& \left.+\left(\frac{\partial \bar{u}}{\partial y}+\frac{\partial \bar{v}}{\partial x}\right)^{2}+\left(\frac{\partial \bar{u}}{\partial z}+\frac{\partial \bar{w}}{\partial x}\right)^{2}+\left(\frac{\partial \bar{v}}{\partial z}+\frac{\partial \bar{w}}{\partial y}\right)\right] \\
& +\frac{\mu}{\bar{T}}\left[2\left\{\overline{\left(\frac{\partial u^{\prime}}{\partial x}\right)^{2}}+\overline{\left(\frac{\partial v^{\prime}}{\partial y}\right)^{2}}+\overline{\left(\frac{\partial w^{\prime}}{\partial z}\right)^{2}}\right\}\right. \\
& \left.+\overline{\left(\frac{\partial u^{\prime}}{\partial y}+\frac{\partial v^{\prime}}{\partial x}\right)^{2}}+\overline{\left(\frac{\partial u^{\prime}}{\partial z}+\frac{\partial w^{\prime}}{\partial x}\right)^{2}}+\overline{\left(\frac{\partial v^{\prime}}{\partial z}+\frac{\partial w^{\prime}}{\partial y}\right)}\right]
\end{aligned}
$$

Similarly, the time-averaged local entropy generation by heat transfer reads:

$$
\begin{aligned}
& \overline{\left(\frac{\Phi_{\Theta}}{T^{2}}\right)}=\frac{\lambda}{\bar{T}^{2}}\left[\left(\frac{\partial \bar{T}}{\partial x}\right)^{2}+\left(\frac{\partial \bar{T}}{\partial y}\right)^{2}+\left(\frac{\partial \bar{T}}{\partial z}\right)^{2}\right] \\
& +\frac{\lambda}{\bar{T}^{2}}\left[\overline{\left(\frac{\partial T^{\prime}}{\partial x}\right)^{2}}+\overline{\left(\frac{\partial T^{\prime}}{\partial y}\right)^{2}}+\overline{\left(\frac{\partial T^{\prime}}{\partial z}\right)^{2}}\right]
\end{aligned}
$$

It can be seen that the fluctuating terms occupies a certain proportion in the time-averaged local entropy generation, which is easily neglected in previous study.

\section{Four local entropy generation rate terms}

According to the time-averaged local entropy generation by dissipation and heat transfer, the entropy generation divided into four terms: local entropy generation by dissipation with mean and fluctuating velocity field and heat flux in a mean and fluctuating temperature field respectively. They are

$$
\begin{aligned}
& S_{\overline{\Phi_{m}}}=\frac{\mu}{\bar{T}}\left[2\left\{\left(\frac{\partial \bar{u}}{\partial x}\right)^{2}+\left(\frac{\partial \bar{v}}{\partial y}\right)^{2}+\left(\frac{\partial \bar{w}}{\partial z}\right)^{2}\right\}\right. \\
& \left.+\left(\frac{\partial \bar{u}}{\partial y}+\frac{\partial \bar{v}}{\partial x}\right)^{2}+\left(\frac{\partial \bar{u}}{\partial z}+\frac{\partial \bar{w}}{\partial x}\right)^{2}+\left(\frac{\partial \bar{v}}{\partial z}+\frac{\partial \bar{w}}{\partial y}\right)\right]
\end{aligned}
$$

$$
\begin{gathered}
S_{\overline{\Phi_{f}}}=\frac{\mu}{\bar{T}}\left[2 \left\{\overline{\left(\frac{\partial u^{\prime}}{\partial x}\right)^{2}}+\overline{\left(\frac{\partial v^{\prime}}{\partial y}\right)^{2}}+\overline{\left.\left(\frac{\partial w^{\prime}}{\partial z}\right)^{2}\right\}}\right.\right. \\
+\overline{\left(\frac{\partial u^{\prime}}{\partial y}+\frac{\partial v^{\prime}}{\partial x}\right)^{2}}+\overline{\left(\frac{\partial u^{\prime}}{\partial z}+\frac{\partial w^{\prime}}{\partial x}\right)^{2}}+\overline{\left.\left(\frac{\partial v^{\prime}}{\partial z}+\frac{\partial w^{\prime}}{\partial y}\right)\right]} \\
S_{\overline{\Phi_{\Theta m}}}=\frac{\lambda}{\bar{T}^{2}}\left[\left(\frac{\partial \bar{T}}{\partial x}\right)^{2}+\left(\frac{\partial \bar{T}}{\partial y}\right)^{2}+\left(\frac{\partial \bar{T}}{\partial z}\right)^{2}\right] \\
S_{\overline{\Phi_{\Theta f}}}=\frac{\lambda}{\bar{T}^{2}}\left[\overline{\left(\frac{\partial T^{\prime}}{\partial x}\right)^{2}}+\overline{\left(\frac{\partial T^{\prime}}{\partial y}\right)^{2}}+\overline{\left(\frac{\partial T^{\prime}}{\partial z}\right)^{2}}\right]
\end{gathered}
$$

\section{NUMERICAL MODELING}

In order to overcome the insufficient modeling stress of the DES method, Spalart et al. (Spalart, Deck, et al., 2006) upgraded the DES method to Delay Detached Eddy Simulation (DDES) method, and others proposed a lowdissipation model for modeling (Su, Yamamoto, et al., 2013. Su, Sasaki, et al., 2013. Guo, Zhang, et al., 2016). The DDES method uses a similar equation to the Shear Stress Transport (SST) model proposed by Menter (Menter, 1994) to limit the length scale in the DES method. It can ensure that the effect of gird is reduced when switching from (Reynolds Average Navier-Stokes) RANS mode to (Large Eddy Simulation) LES mode. The DDES method is constructed by modifying the parameter $r$ in S-A model as below:

$$
r_{d}=\frac{v+v_{t}}{\left(U_{i, j} U_{i, j}\right)^{0.5} \kappa^{2} d^{2}}
$$

where $U i_{, j}$ is the velocity gradient, $\kappa$ is the constant parameter $(\kappa=0.41), d$ is the distance from the closest wall, $v$ is the kinematic viscosity. With this modification, this parameter can be applied to any eddy viscosity model. The parameter $r_{d}$ is mainly used to construct the following restriction equation:

$$
f_{d}=1-\tanh \left(\left[8 r_{d}\right]^{3}\right), \tilde{d}=d-f_{d} \max \left(0, d-C_{D E S} \Delta\right) .
$$

where the $C_{D E S}$ is $0.65, \Delta$ is the maximum length scale of mesh element. This equation can effectively limit the RANS and LES gray transition zone (Spalart, Deck, et al., 2006), overcoming the problem of insufficient modeling stress.

\section{COMPUTATIONAL SETUP AND APPROACH}

In this paper, the linear rotor in a single stage axial flow turbine is selected as the research object. The computational domain and mesh distribution are shown in Figure 1(a) and 1(b). The computational domain is mainly divided into three mesh blocks as inlet, blade and outlet blocks. The length of the inlet block is the same as the axial chord length, and the 
outlet block is twice the length of the axial chord, which can ensure that the downstream flow is fully developed.

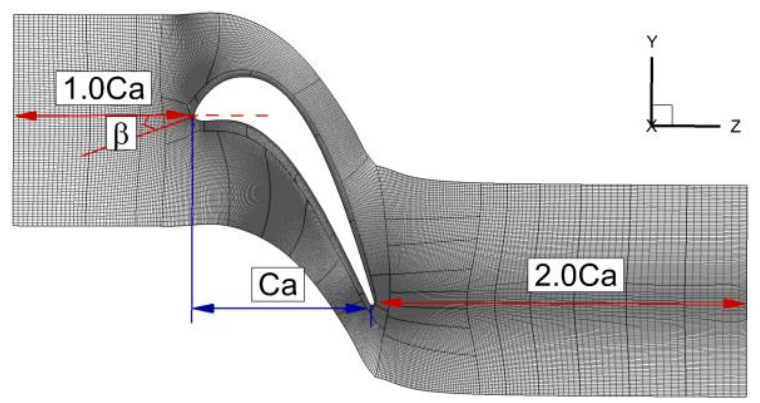

(a) Computational domain

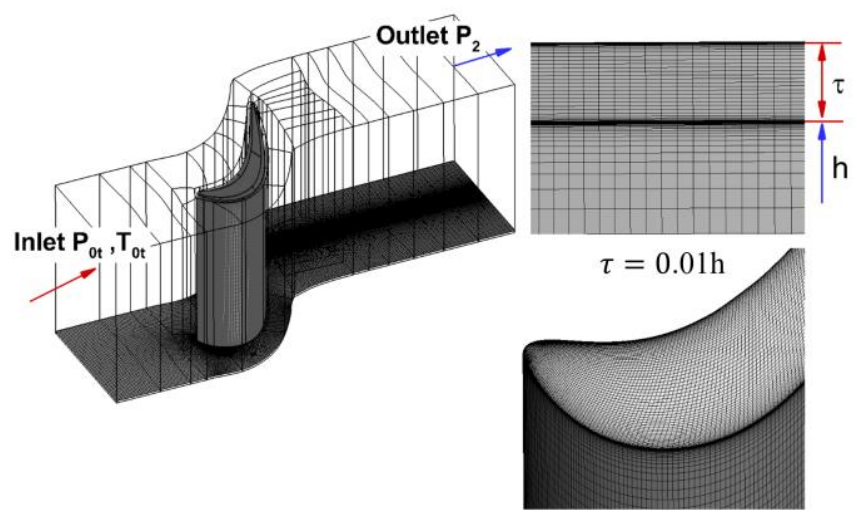

(b) Boundary conditions and mesh details

Figure 1 The mesh and blocks distribution

Commercial software NUMECA AutoGrid5 is used to generate a $4 \mathrm{HO}$ block structured mesh, and the mesh quality in the tip region is finely tuned for high quality. The overall mesh is about 9.11 million points, with 53 layers of mesh in the clearance and 113 layers along the spanwise. The minimum orthogonality among all mesh block is 62.1 degrees, and the maximum expansion ratio is 1.43 . The maximum $y+$ of the first layer off-wall mesh is 0.156 , which ensures a good resolution of the viscous sublayer. Away from the wall, the mesh element is nearly isotropic, with $\Delta x^{+} \approx 110$ and $\Delta z^{+} \approx 20$, which are in agreement with the recommended values for LES predictions (Javier and Parviz, 2006). In order to accelerate the calculation, the grid is split into 58 blocks, with an average of 0.16 million points per block.

The inlet total temperature, $289 \mathrm{~K}$ and the inlet total pressure, $100819 \mathrm{~Pa}$ with flow angle are imposed. The static pressure, $97080 \mathrm{~Pa}$ is determined at the outlet. The inlet Reynolds number is 230000 . The maximum Mach number in this case is less than 0.3 , so the compressibility effects are neglected in the computation. No-slip and adiabatic boundary conditions are set to all cascade walls. The periodic boundary condition is used at pitch-wise boundary. The cascade is modelled as a stationary endwall, and the influence of the relative motion is an on-going research.

An in-house MPI-parallel multi-block structured finite volume code (Su and Yuan, 2010) is used in this work. The governing equation of this code is the integral form of the Navier-Stokes equation as below

$$
\frac{\partial}{\partial \tau} \iiint U d V+\frac{\partial}{\partial t} \iiint U d V+\oint\left(F_{c}-F_{v}\right) \cdot n d S=0 .
$$

The first term on the left side of the equation represents the pseudo time term. In the time marching solution, the three stage Runge-Kutta/implicit scheme and the multigrid method are used. The second term represents the physical time term, which controls the unsteady evolution. In unsteady calculation, dual-timestep of an optimized, secondorder, backward difference temporal scheme (BDF2opt) method is adopted. In this work, in order to reduce the numerical dissipation, a high-accuracy 5th order Weighted Essentially non-oscillatory (WENO) reconstruction scheme (Su, Yamamoto, et al., 2013. Su, Sasaki, et al., 2013. Wang, Lin, et al., 2017) is adopted and the modified Roe scheme with low Mach number preconditioning ( $\mathrm{Li}$ and $\mathrm{Li}, 2016$ ) is used to compute the convective flux. 4th order centered scheme is used to discretize the viscous term. In the turbulence equation, the source term of the Spalart-Allmaras equation dominates and the discretization methods for the convective and viscous terms have a marginal effect. For stability, a second order upwind method and second order centered scheme are used for the convective and viscous terms in the turbulence model, respectively.

Detailed validation and resolution about DDES method have been conducted by before work ( $\mathrm{Li}, \mathrm{Su}$, et al., 2019). Results show that the less than $20 \%$ of total turbulent kinetic energy is modeled by LES expect for the near wall area where RANS works. It indicates that the leakage vortex region is mostly resolved by LES.

\section{RESULTS AND DISCUSSION}

\section{Tip leakage flow process analysis}

At the blade tip, due to the pressure difference between the suction side and the pressure side, the fluid flows from the pressure side to the suction side. Then the complex vortical structures inside and around the tip are formed. Figure 2 shows the vortical structures near tip gap. The black streamlines are the flows from pressure side to suction side, which denotes the tip leakage vortex (TLV). The red streamlines are the tip leakage flows inside the tip gap, which leads to separation vortex (SV). The flows from the suction side lead to the passage secondary vortex (PSV). It can be found that the tip leakage vortex forms at $25 \%$ of axial chord, and the separation vortex forms from the leading edge to trailing edge. As the tip leakage flow forming, a portion of the passage secondary vortex moves beneath the tip leakage vortex, and the other portion interacts with the tip leakage vortex. Some separation vortex in tip gap flows from pressure side to suction side and interacts with tip leakage vortex and passage flow secondary vortex. Finally, the three vortical structures interact together and move downstream. 


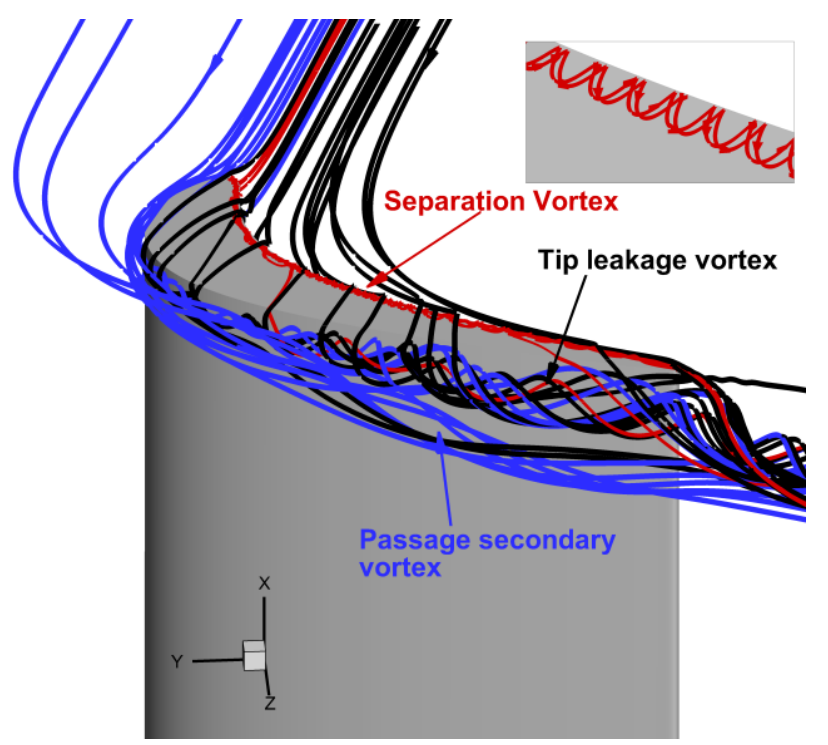

Figure 2 Time-averaged velocity streamlines for tip leakage flow

Figure 3 shows projection of the vorticity vector $\nabla \times u$ onto the main flow direction $u /|u|$. It shows that at $25 \% \mathrm{Ca}$, in the tip clearance, there is separation vortex near the pressure side. This separation vortex reduces the flow area in tip clearance. As moving downstream, the pressure difference between the suction side and the pressure side gradually increases, so that the fluid on the pressure side has more kinetic energy passing through the tip clearance, and then the tip leakage vortex forms at the suction side. Since the energy of the tip vortex is lower in the upstream, the passage secondary vortex cannot be completely moved by the leakage vortex. As the leakage vortex continues to grow up and develop, the leakage vortex splits the passage secondary vortex into several parts. The small scale passage secondary vortices dissipated and the main vortex core of passage secondary vortex is under the tip leakage vortex, which is smaller than tip leakage vortex. As the tip leakage vortex going downstream, the passage secondary vortex and the leakage vortex gradually merge together and dissipate.
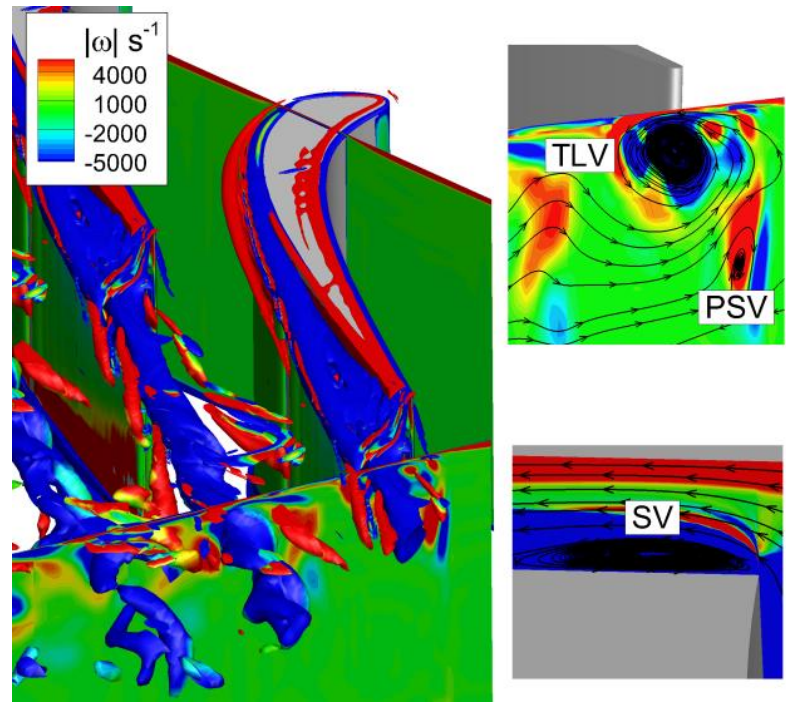

Figure 3 Instantaneous leakage flow process (surfaces of the vorticity contour).

\section{Entropy generation analysis}

As mentioned before, loss coefficients are generally used to evaluate the flow loss in turbine design, such as energy loss coefficient, enthalpy loss coefficient, and total pressure loss coefficient. However, these loss coefficients are only a global value, which is the result of sum of losses, and it cannot provide information about local losses in flow field. The rich numerical simulation results of the flow field now make it possible to measure or visualize the factors which cannot be measured or visualized before. Therefore, the loss coefficient and the loss source can be directly linked together, which is very advantageous for studying the loss mechanism. According to the entropy transportation equation, the local entropy generation is a reasonable quantitative measure of irreversibility loss. So the case of tip leakage flow loss is investigated by local entropy generation. Since the previous study is found that the local entropy generation by dissipation is almost the main source of loss, this work mainly investigates the dissipation part.

\section{Total local entropy generation by dissipation}

Since the interaction between tip leakage vortex and passage secondary vortex is strong at $110 \%$ of axial chord, this section is selected as the tip leakage flow loss analysis. Figure 4 shows the total local entropy generation by dissipation at $110 \%$ of axial chord, and it is the section above the half spanwise. The vertical axis is the spanwise direction and the horizontal axis is the pitchwise direction. Referring to Figure 3 and Figure 4, the loss by dissipation mainly occurs at this place where the tip leakage vortex interacts with the passage vortex and the endwall. The interaction between tip leakage vortex and passage secondary vortex leads to large deformation, so the loss occurred here is greater than tip leakage vortex and passage secondary vortex itself. Due to the boundary effect on the endwall, the total local entropy generation is also large.

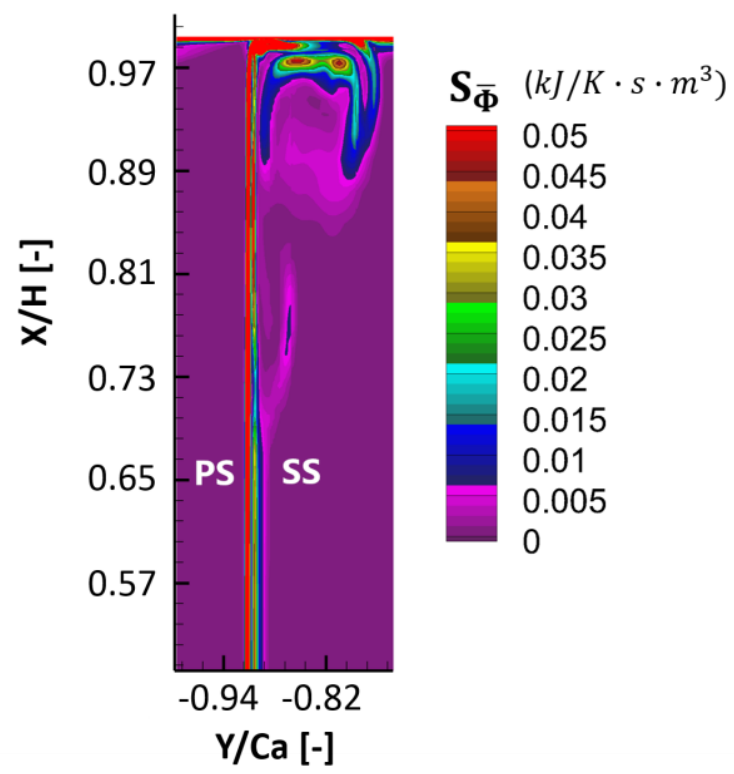

Figure 4 The local entropy generation distribution by dissipation at $110 \%$ of axial chord 


\section{Mean local entropy generation by dissipation}

Figure 5 shows the mean local entropy generation by dissipation, and this entropy generation is produced by mean velocity field. It can be seen that the distribution of mean local entropy generation is similar to the local entropy generation rate, and the loss occurs at tip leakage vortex and passage secondary vortex and their interaction place, and endwall. The main cause of the loss happens on where the tip leakage vortex and passage secondary vortex interacts with each other and the endwall. However, loss at the center of the vortex core of the tip leakage vortex and passage secondary vortex is relatively small. Because the kinetic energy in the vortex core is large and the deformation is small, so the loss caused by dissipation of mean velocity field is small. It can be found that the mean and total entropy generation by dissipation belong to the same magnitude. In turbulent velocity field, the mean local entropy generation by dissipation is the main source of total local entropy generation.

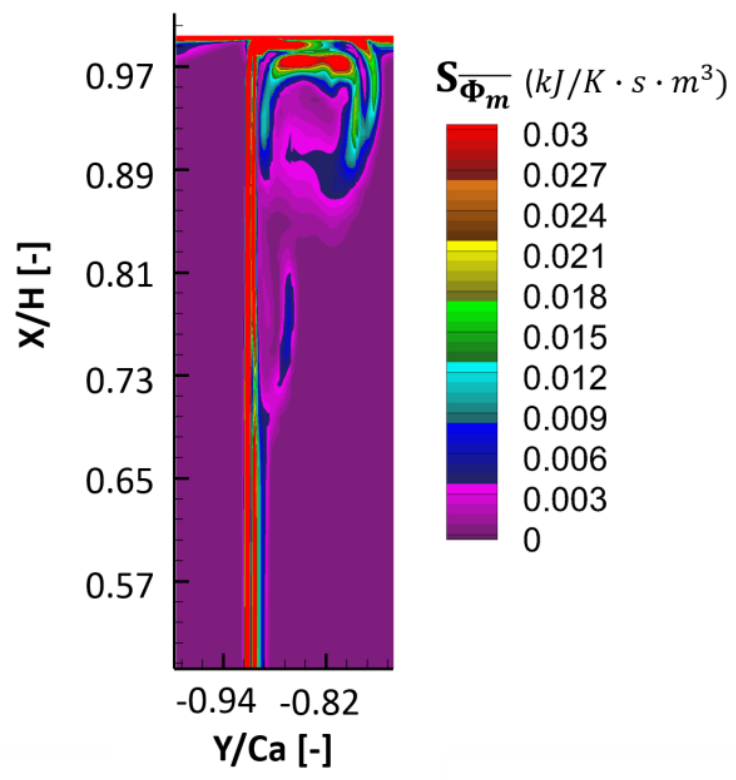

Figure 5 The mean local entropy generation distribution by dissipation at $110 \%$ of axial chord

\section{Fluctuating local entropy generation by dissipation}

From Figure 6, it shows the fluctuating local entropy generation distribution, and this entropy generation is produced by fluctuating velocity field. It can be seen that the quantity of fluctuating local entropy generation by dissipation is smaller than mean local entropy generation. The fluctuating influence also increases the local entropy generation in the flow field, but the range of its influence is smaller than the mean flow influence. The increase loss of fluctuating occurs where the tip leakage vortex and passage secondary vortex interacts most strongly, and the endwall has little increase loss due to fluctuating.

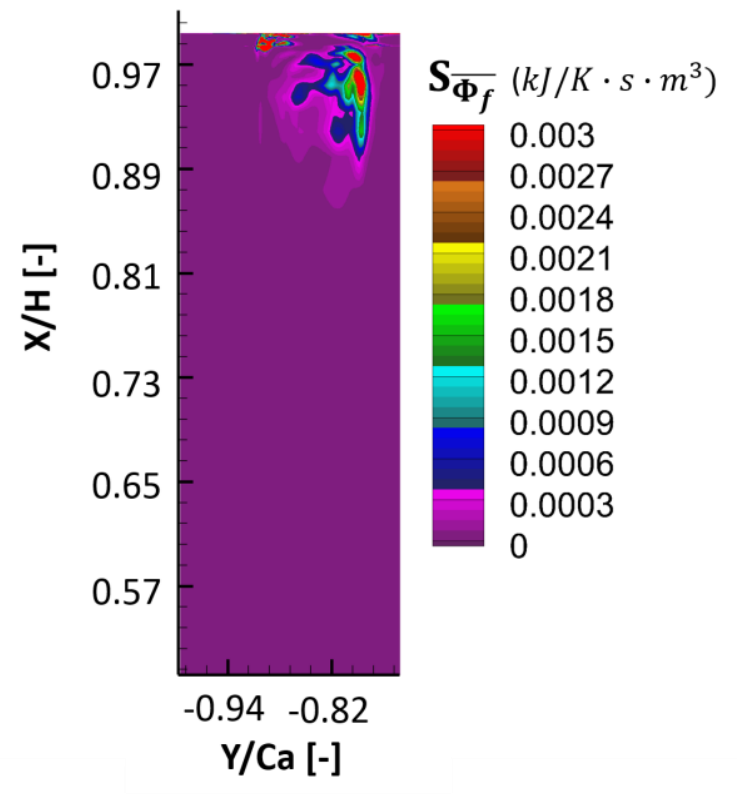

Figure 6 The fluctuating local entropy generation distribution by dissipation at $110 \%$ of axial chord

\section{Comparison between different local entropy generation rates.}

Figure 7 shows the area average of different local entropy generation by dissipation. It can be seen that the mean local entropy generation is main source of total local entropy generation, and the fluctuating local entropy generation is extremely less. It means that the mean flow plays a key role to the overall loss of flow field.

It can be seen from Figure 6 and Figure 7 that the amount of fluctuating local entropy generation is much smaller than mean local entropy generation. From Figure 8, it can be found that the largest fluctuating local entropy generation rate is about $30 \%$ of total local entropy generation. This shows that the fluctuating effect is important to increase the local entropy generation, and the loss caused by influence of fluctuating cannot be ignored.

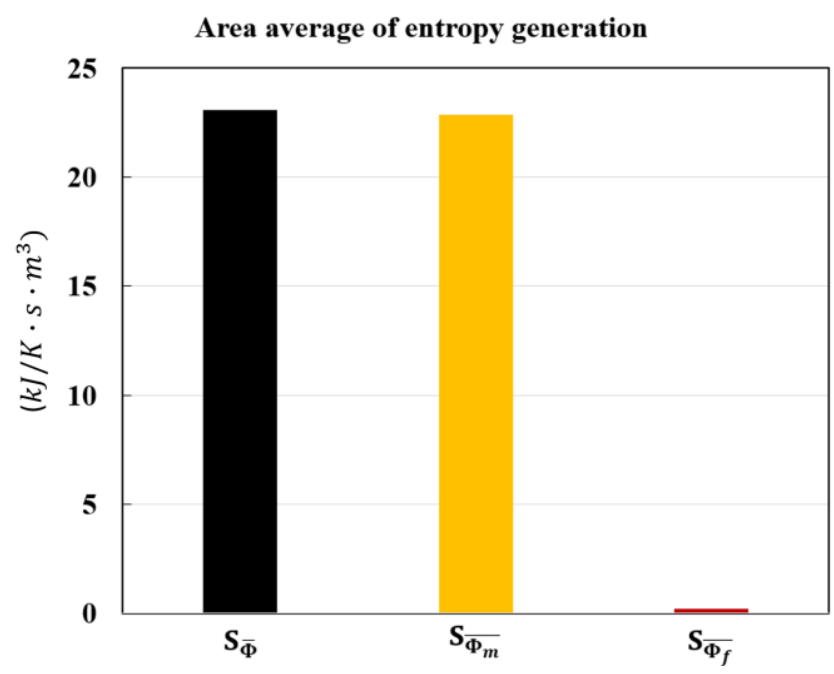

Figure 7 The different local entropy generation distribution by dissipation at $110 \%$ of axial chord 


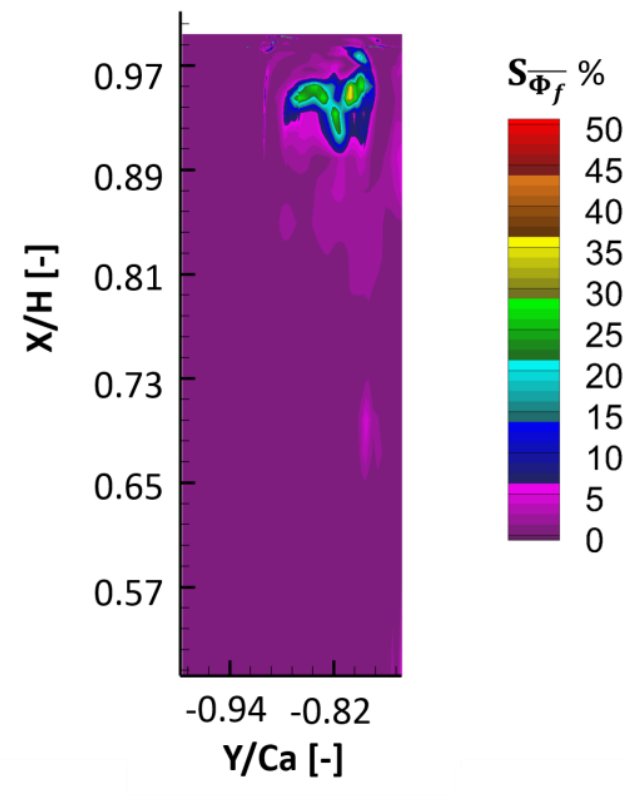

\section{Figure 8 The fluctuating local entropy generation rate distribution by dissipation at $110 \%$ of axial chord}

\section{CONCLUSIONS}

In this work, based on entropy transportation equation, the time-averaged entropy generation is devised. The high fidelity calculation method DDES is used to simulate a unshrouded blade, and the tip leakage flow is analysed by the entropy generation rate. The conclusions are as follows:

In complex flow field, DDES method can obtain a rich flow field. In this case, DDES method captures the development and interaction process of the tip leakage flow and the secondary flow. Which is very helpful for the analysis of tip leakage flow mechanism in the flow field.

The time-averaged entropy generation includes four terms: dissipation in a mean and fluctuating velocity field and heat flux in a mean and fluctuating temperature field respectively. The mean local entropy generation is main source of total local entropy generation, and the fluctuating part is less. However, the largest local entropy generation rate caused by fluctuating is about $30 \%$ of the total entropy generation, which indicates that the impact of fluctuating on loss cannot be neglected.

\section{ACKNOWLEDGMENTS}

This study is supported by the National Natural Science Foundation of China (Project Grant No. 51876098) and National Science and Technology Major Project (2017-III0009-0035), also supported by the Open fund from State Key Laboratory of Aerodynamics.

\section{References}

Bejan A., Kestin J. (1983). Entropy generation through heat and fluid flow, 50(2):475-0.

Bian X T, Lin D, Su X R, et al. (2017). Study of Shock Wave-Boundary Layer Interaction Mechanism in a Transonic
Vane Based on DDES. Journal of Propulsion Technology, 38(10):2254-2261.

Chu S X., Liu L H. (2009). Entropy generation analysis of two-dimensional high-temperature confined jet. International Journal of Thermal Sciences, 48(5):998-1006.

Denton,J.D. (1993). Loss mechanisms in turbomachines. Journal of turbomachinery; (United States). 115:4. 10.1115/93-GT-435.

Gou, J., Zhang, Y., Su, X., and Yuan, X. (2016). "Numerical investigation on the effects of real industrial bleeding geometry in a high-speed compressor stage". Journal of Mechanical Science \& Technology, 30(11), pp. 5275-5286.

Jin Y., Herwig H. (2014). Turbulent flow and heat transfer in channels with shark skin surfaces: Entropy generation and its physical significance. International Journal of Heat and Mass Transfer, 70:10-22.

Javier, J., and Parviz, M. (2006). The minimal flow unit in near-wall turbulence. Journal of Fluid Mechanics, 225(1), pp. 213-240.

Kock F., Herwig H. (2004). Local entropy production in turbulent shear flows: a high-Reynolds number model with wall functions. International Journal of Heat and Mass Transfer, 47(10-11):2205-2215.

Kock F., Herwig H. (2005). Entropy production calculation for turbulent shear flows and their implementation in cfd codes. International Journal of Heat \& Fluid Flow, 26(4):672-680.

Li, Hui \& Su, Xinrong \& Yuan, Xin. (2019). Entropy Analysis of the Flat Tip Leakage Flow with Delayed Detached Eddy Simulation. Entropy. 21. 10.3390/e21010021.

Lee M, Malaya N, Moser R D. (2015). Direct numerical simulation of turbulent channel flow up to $\operatorname{Re} \tau \approx 5200$. Journal of Fluid Mechanics, 774(4):395-415.

Lin D, Su X R, Yuan X. (2016). DDES Simulation of a High-Pressure Turbine Guide Vane. Journal of Engineering Thermophysics, V37(10):2084-2088.

Lin, D., Yuan, X., and Su, X. (2017). Local entropy generation in compressible flow through a high pressure turbine with Delayed Detached Eddy Simulation. Entropy, 19(1), p. 29.

Li, X., and Li, X. (2016). All-speed Roe scheme for the large eddy simulation of homogeneous decaying turbulence. International Journal of Computational Fluid Dynsmics, 30(1), pp. 69-78.

Menter, F. R. (1994). Two-equation eddy-viscosity turbulence models for engineering applications. AIAA Journal, 32(8), pp. 1598-1605.

Su X R, Yuan X. (2016). Adaptive Mesh Refinement for Complex Turbomachinery Flow. Journal of Engineering Thermophysics, V37(2):259-263.

Su X, Xin Y. (2010). Implicit solution of time spectral method for periodic unsteady flows. International Journal for Numerical Methods in Fluids, 63(7):860-876.

Su, X., Yamamoto, S., and Yuan, X. (2013). On the accurate prediction of tip vortex: effect of numerical schemes. In Proceedings of ASME Turbo Expo 2013. 
Su, X., Sasaki, D., and Nakahashi, K. (2013). On the efficient application of Weighted Essentially Nonoscillatory scheme. International Journal for Numerical Methods in Fluids, 71(2), pp. 185-207.

Spalart, P. R., Deck, S., Shur, M. L., Squires, K. D., Strelets, M. K., and Travin, A. (2006). A new version of Detached-eddy Simulation, resistant to ambiguous grid densities. Theoretical \& Computational Fluid Dynamics, 20(3), p. 181.

Wang, H., Lin, D., Su, X., and Yuan, X. (2017). Entropy

analysis of the interaction between the corner separation and wakes in a compressor cascade. Entropy, 19(7).

Yang G, Yao Y, Fang J, et al. (2016). Large-eddy simulation of shock-wave/turbulent boundary-layer interactions and its control using Sparkjet. He Sixth International Symposium on Physics of Fluids. World Scientific Publishing Company, 617-629. 